\title{
Recomendação de Ações Pedagógicas Utilizando Planejamento Automático e Taxonomia Digital de Bloom
}

\author{
Newarney T. Costa $^{1}$, Cleon X. Pereira Junior ${ }^{1}$, Márcia A. Fernandes ${ }^{2}$ \\ ${ }^{1}$ Instituto Federal Goiano (IF Goiano) - Iporá - GO - Brasil \\ ${ }^{2}$ Faculdade de Computação - Universidade Federal de Uberlândia (UFU) \\ Uberlândia - MG - Brasil \\ \{newarney.costa,cleon.junior\}@ifgoiano.edu.br, marcia@ufu.br
}

\begin{abstract}
This paper presents an approach for the recommendation of pedagogical actions in Virtual Learning Environment (VLE), using automatic planning. The actions considered by the planner are structured by Bloom's Digital Taxonomy. The state is specified according to the student model. This model contains information provided by the VLE and the ASSIST questionnaire. A base of cases containing plans associated with student models is maintained and initially consulted in order to optimize the process. A proof of concept shows the feasibility of using Automated Planning effectively in the context of recommendations for generic pedagogical actions.
\end{abstract}

Resumo. Este trabalho apresenta uma abordagem para recomendação de ações pedagógicas em Ambiente virtual de Aprendizagem (AVA), utilizando planejamento automático. As ações consideradas pelo planejador são estruturadas pela Taxonomia Digital de Bloom. O estado é especificado de acordo com o modelo do estudante. Esse modelo é composto por metadados gerados a partir de AVA e informações coletadas através do questionário ASSIST. Na abordagem existe uma base de casos contendo planos associados a modelos de estudantes com a finalidade de otimizar o processo. Uma prova de conceito mostra a viabilidade da utilização do Planejamento Automático de maneira efetiva no contexto de recomendações de ações pedagógicas genéricas.

\section{Introdução}

A recomendação pedagógica personalizada, no contexto de e-learning, frequentemente conta com técnicas de Inteligência Artificial (IA) no intuito de melhorar a aprendizagem [Xu et al. 2014]. Observa-se na literatura que diversas características podem ser utilizadas para compor os parâmetros necessários à geração de recomendações pedagógicas personalizadas, como por exemplo, emoções, perfis de personalidade e inteligências múltiplas, metadados de ambientes virtuais, entre outros [Dantas et al. 2018].

A técnica de Planejamento em Inteligência Artificial ou Planejamento Automatizado (PA) consiste em definir, de forma automatizada, uma sequência de ações baseadas no cumprimento de um objetivo. Portanto, espera-se que nesse processo ocorra a otimização na escolha das ações, de modo a alcançar efetivamente o objetivo esperado. Diante disso, existe uma variedade de planejadores e formas distintas de classificá-los [Nau et al. 2004]. A modelagem do processo de aprendizagem sob esta perspectiva é viável e também um desafio emergente [Caputi and Garrido 2015]. 
VIII Congresso Brasileiro de Informática na Educação (CBIE 2019)

Anais do XXX Simpósio Brasileiro de Informática na Educação (SBIE 2019)

Com a finalidade de expor um modelo para recomendação de ações pedagógicas, baseado em PA, que utiliza técnicas para identificação do perfil cognitivo do estudante, além de ações pedagógicas relacionadas à evolução do processo cognitivo, este artigo foi assim organizado: Na Seção 2, são abordados os trabalhos que utilizam técnicas de PA para personalização de alguma etapa do processo pedagógico dando ênfase às ações pedagógicas e ao modelo do estudante; Na Seção 3 é apresentada a proposta para recomendação de ações pedagógicas por meio de PA, baseada na TDB e no ASSIST; Na Seção 4 são discutidas as considerações acerca dessa abordagem.

\section{Uso de PA no Contexto Educacional}

No contexto do ensino personalizado, o PA é utilizado para otimização de etapas do processo de ensino-aprendizagem. Uma de suas principais vantagens repousa no fato de que esse processo pode ser modelado adequadamente como um problema de PA, pois as características atuais de um estudante podem ser compreendidas como seu estado inicial e seu objetivo pode ser percebido como o estado no qual esse deseja que suas características evoluam [Costa et al. 2019]. A Tabela 1 apresenta uma síntese de nove trabalhos que utilizam PA para automatização de etapas do processo de ensino-aprendizagem.

O PA baseado em regras de associação foi utilizado em três trabalhos mostrados na Tabela 1. Em [Garrido and Onaindia 2013] e [Caputi and Garrido 2015], esta técnica tem como objetivo geral o sequenciamento de atividades personalizadas para cada estudante, por meio da seleção adequada de Objetos de Aprendizagem (OA). Em [Marinov and Valova 2016], o foco principal foi a implementação de uma estratégia de ensino global e individualizada para o estudante.

O Planejamento Baseado em Casos (PBC) foi utilizado em [Garrido and Morales 2014, Garrido et al. 2016] para personalizar a ordem em que os OA são ofertados ao estudante. Como uma das vantagens do PBC, esse trabalho aponta ainda que a partir dos caminhos de aprendizagem mapeados numa base de casos, previamente planejados, é possível reutilizá-los para outros estudantes com perfis semelhantes, aumentando assim, a eficiência do sistema proposto.

Dois trabalhos exibidos na Tabela 1 exploraram a utilização do planejamento hierárquico no contexto de e-learning. Em [Pireva and Kefalas 2018] esta técnica foi utilizada com o intuito de definir uma sequência de OA correspondentes ao conhecimento prévio de um aprendiz e ao seu objetivo. Em [Limongelli and Sciarrone 2014], esse foi utilizado para auxiliar o estudante durante uma atividade de aprendizagem, por meio do conhecimento prévio de seu estado cognitivo, de seu estilo de aprendizagem e da estratégia didática do professor. O space-state planning foi utilizado em [Sanchez Nigenda et al. 2017], por ser considerado adequado para a representação de currículos de aprendizagem, e com a finalidade de relaxar as restrições totais de ordenação dos caminhos de aprendizagem, utilizou-se a programação matemática.

Diante do exposto, observa-se uma variedade de técnicas de PA que foram aplicadas no contexto de e-learning, com o intuito de automatizar a personalização de uma ou mais etapas do processo pedagógico. Nota-se ainda que a utilização de características adequadas do estudante acerca do contexto no qual o planejador atuará é de suma importância para a utilização efetiva do PA. Dentre as técnicas utilizadas, o PBC apresenta como uma de suas principais vantagens, a melhora na eficiência do sistema, pois evita muitas vezes 
VIII Congresso Brasileiro de Informática na Educação (CBIE 2019)

Anais do XXX Simpósio Brasileiro de Informática na Educação (SBIE 2019)

Tabela 1. Trabalhos que utilizam PA no contexto educacional.

\begin{tabular}{|c|c|c|c|}
\hline Artigo & $\begin{array}{l}\text { Modelagem do Estu- } \\
\text { dante }\end{array}$ & $\begin{array}{l}\text { Planejamento } \\
\text { Automático }\end{array}$ & Intervenção Pedagógica \\
\hline $\begin{array}{l}\text { [Garrido and } \\
\text { Onaindia } \\
\text { 2013] }\end{array}$ & $\begin{array}{l}\text { Metadados de AVA; Estilo } \\
\text { de aprendizagem (Felder- } \\
\text { Silverman); Preferências. }\end{array}$ & $\begin{array}{l}\text { Regras r de } \\
\text { associação; Lógica } \\
\text { temporal. }\end{array}$ & $\begin{array}{l}\text { Geração de rota de aprendizagem } \\
\text { personalizada com o objetivo de } \\
\text { oferecer cursos personalizados. }\end{array}$ \\
\hline $\begin{array}{l}\text { [Garrido and } \\
\text { Morales 2014] }\end{array}$ & $\begin{array}{l}\text { Metadados de AVA; Estilo } \\
\text { de Aprendizagem (Felder- } \\
\text { Silverman). }\end{array}$ & Baseado em casos. & $\begin{array}{l}\text { Geração de rota de aprendizagem } \\
\text { personalizada, por meio do se- } \\
\text { quenciamento de OA. }\end{array}$ \\
\hline $\begin{array}{l}\text { [Limongelli } \\
\text { and Sciarrone } \\
\text { 2014] }\end{array}$ & $\begin{array}{l}\text { Estado cognitivo (Taxono- } \\
\text { mia de Bloom); Estilo } \\
\text { de aprendizagem (Felder- } \\
\text { Silverman). }\end{array}$ & $\begin{array}{l}\text { Hierárquico; } \\
\text { Lógica Temporal } \\
\text { Linear. }\end{array}$ & $\begin{array}{l}\text { Geração de avaliações personali- } \\
\text { zadas, por meio da oferta de hi- } \\
\text { permídia adaptativa. }\end{array}$ \\
\hline $\begin{array}{l}\text { [Caputi and } \\
\text { Garrido 2015] }\end{array}$ & $\begin{array}{l}\text { Metadados de AVA; Estilo } \\
\text { de aprendizagem (Felder- } \\
\text { Silverman). }\end{array}$ & $\begin{array}{l}\text { Regras } \\
\text { associação. }\end{array}$ & $\begin{array}{l}\text { Criação de rota de aprendizagem } \\
\text { personalizada, por meio do se- } \\
\text { quenciamento de OA. }\end{array}$ \\
\hline $\begin{array}{l}\text { [Torres and } \\
\text { Guzmán-Luna } \\
\text { 2015] }\end{array}$ & $\begin{array}{l}\text { Estilo de aprendizagem } \\
\text { (Honey-Alonso). }\end{array}$ & Reativo. & $\begin{array}{l}\text { Oferta de rotas de aprendizagem } \\
\text { adaptadas ao perfil do estudante, } \\
\text { por meio da seleção OA. }\end{array}$ \\
\hline $\begin{array}{l}\text { [Garrido et al. } \\
\text { 2016] }\end{array}$ & $\begin{array}{l}\text { Metadados de AVA; Estilo } \\
\text { de Aprendizagem (Felder- } \\
\text { Silverman); Preferências. }\end{array}$ & Baseado em casos. & $\begin{array}{l}\text { Geração de rota de aprendizagem } \\
\text { personalizada por meio de uma } \\
\text { sequência de OA. }\end{array}$ \\
\hline $\begin{array}{l}\text { [Marinov and } \\
\text { Valova 2016] }\end{array}$ & $\begin{array}{l}\text { Análise do conhecimento } \\
\text { do estudante sobre o } \\
\text { conteúdo. }\end{array}$ & $\begin{array}{l}\text { Regras } \\
\text { associação. }\end{array}$ & $\begin{array}{l}\text { Personalização de cursos, por } \\
\text { meio da oferta de exercícios base- } \\
\text { ados no perfil do estudante. }\end{array}$ \\
\hline $\begin{array}{l}\text { [Sanchez } \\
\text { Nigenda et al. } \\
\text { 2017] }\end{array}$ & Não discutido. & Hierárquico. & $\begin{array}{l}\text { Geração de rota de aprendiza- } \\
\text { gem personalizada, por meio da } \\
\text { composição de OA. }\end{array}$ \\
\hline $\begin{array}{l}\text { [Pireva and } \\
\text { Kefalas 2018] }\end{array}$ & $\begin{array}{l}\text { Metadados de AVA; Estilo } \\
\text { de aprendizagem (Felder- } \\
\text { Silverman); Taxonomia de } \\
\text { Bloom; Preferências. }\end{array}$ & Hierárquico. & $\begin{array}{l}\text { Geração de rota de aprendizagem } \\
\text { personalizada, por meio do se- } \\
\text { quenciamento de OA. }\end{array}$ \\
\hline
\end{tabular}

que o sequenciamento de ações para casos similares seja realizado novamente, já que este é um processo caro computacionalmente.

Outra questão relevante diz respeito às ações pedagógicas adotadas nos trabalhos propostos. Geralmente estas baseiam-se em conteúdos específicos, por meio do sequenciamento de OA. Tal estratégia apresenta vantagens sob o ponto de vista da simplificação das ações pedagógicas recomendadas, entretanto, não permite explorar o processo pedagógico em si, pois geralmente foca em recomendação de conteúdo. Portanto, a proposição de alternativas cujo foco seja o processo cognitivo do estudante, apresenta-se como um desafio que pode contribuir para o avanço na área de informática na educação.

\subsection{Ações Pedagógicas}

Nos trabalhos relatados na Tabela 1, é possível observar que as recomendações pedagógicas oriundas do PA, majoritariamente focam em sequenciamento de OA, requerendo do ambiente no qual estas atuarão um conhecimento muito específico acerca da estrutura curricular de cursos, bem como seu conteúdo. Diante disso, nota-se uma carência 
VIII Congresso Brasileiro de Informática na Educação (CBIE 2019)

Anais do XXX Simpósio Brasileiro de Informática na Educação (SBIE 2019)

da exploração de técnicas cujo foco seja a recomendação de ações pedagógicas genéricas, contemplando o processo pedagógico e não o conteúdo.

A Taxonomia de Bloom (TB) estrutura os objetivos educacionais em três domínios: cognitivo; afetivo; e psicomotor. Uma revisão amplamente utilizada da TB, denominada Taxonomia Revisada de Bloom (TRB) aponta que o domínio cognitivo é analisado sob as dimensões do conhecimento e do processo cognitivo [Krathwohl 2002]. Nesta revisão, a TB foi estruturada de modo que os objetivos educacionais sejam descritos como verbos, compondo a dimensão do processo cognitivo. Essa dimensão é composta por seis níveis, como na TB original, porém reordenados e renomeados. Outra dimensão, denominada dimensão do conhecimento, passou a ser composta por quatro níveis e é responsável por possibilitar a representação da intersecção de assuntos. Na Tabela 2 é possível observar esta representação, considerando ambas as dimensões:

Tabela 2. Representação bidimensional da Taxonomia Revisada de Bloom.

\begin{tabular}{|l|l|l|l|l|l|l|}
\cline { 2 - 7 } \multicolumn{1}{c|}{} & \multicolumn{5}{c|}{ Dimensão do Processo Cognitivo } \\
\hline Dim. do Conhecim. & 1. Lembrar & 2. Entender & 3. Aplicar & 4. Analisar & 5. Avaliar & 6. Criar \\
\hline A. Factual & & & & & & \\
\hline B. Conceitual & & & & & & \\
\hline C. Procedural & & & & & & \\
\hline D. Metacognitivo & \multicolumn{3}{l|}{ Fonte: Adaptado de [Krathwohl 2002]. } & & \\
\hline
\end{tabular}

Os objetivos presentes nas categorias das dimensões que compõem o domínio cognitivo, da esquerda para a direita e de cima para baixo, são uma variação das habilidades cognitivas, partindo de Lower Order Cognitive Skills (LOCS), para Higher Order Cognitive Skills (HOCS). Na TB original, as categorias dessas dimensões possuem uma hierarquia em relação à complexidade dos objetivos, porém após sua revisão, considerouse mais relevante a forma como o professor utiliza a TB, permitindo então a flexibilização desta hierarquia, por meio da sobreposição de categorias. Assim, o PA baseado no estado cognitivo do estudante, apresenta-se como uma ferramenta importante na definição de ações necessárias para que o estudante atinja o objetivo esperado. De maneira geral, sua utilização sob o ponto de vista da informática na educação também é explorada, como por exemplo em [Haendchen Filho et al. 2018].

Outra revisão da TB, denominada Taxonomia Digital de Bloom (TDB), vem sendo utilizada em trabalhos recentes, pois representa comportamentos, ações e formas de trabalho emergentes, em virtude dos avanços associados às Tecnologias da Informação e Comunicação (TIC) [Churches 2010]. A TDB associa as categorias presentes na dimensão do processo cognitivo existentes na TRB, à atividades relacionadas com formas como o estudante pode interagir com os recursos digitais disponíveis, sejam eles via Web ou não. Por exemplo, um estudante que se encontre na categoria Lembrar, pode executar atividades como: destacar palavras-chave ou frases em um arquivo digital; marcar ou organizar sites, arquivos ou recursos, para utilizar posteriormente; fazer buscas on-line para recuperar recursos ou conteúdo; entre outros.

Em [de Paiva and Padilha 2012], a TDB é utilizada sob a perspectiva da metodologia WebQuest, com o intuito de melhor guiar o desenvolvimento de tarefas considerando 
VIII Congresso Brasileiro de Informática na Educação (CBIE 2019)

Anais do XXX Simpósio Brasileiro de Informática na Educação (SBIE 2019)

novos recursos ofertados pelo desenvolvimento tecnológico. Em [Ohler 2013], a TDB é apresentada sob o ponto de vista da utilização na alfabetização, por meio do uso de OA adequados para o conto de histórias. Em [Leu et al. 2017], é evidenciada a importância da evolução das técnicas pedagógicas utilizadas devido à realidade proporcionada pela educação mediada por computador. Diante dos trabalhos supracitados, nota-se a possibilidade de utilizar a TDB como estruturação para recomendação de ações pedagógicas por meio de PA, objetivando a evolução processo cognitivo do estudante.

\subsection{Modelo do Estudante}

O modelo do estudante corresponde à estruturação de informações como atributos, preferências, estilos ou estratégias de aprendizagem, entre outros. Na Tabela 1, também é possível observar os elementos utilizados para modelar o perfil estudante. Nos trabalhos apresentados por [Garrido and Onaindia 2013, Garrido and Morales 2014, Limongelli and Sciarrone 2014, Garrido et al. 2016, Pireva and Kefalas 2018] o perfil do estudante foi definido com base no estilo de aprendizagem proposto em [Felder et al. 1988] em conjunto com outras características, como por exemplo: associado aos desejos e necessidades do estudante; em conjunto com os requisitos do curso, impostos por meio das inter-relações entre OA; ou ainda associados à TRB. Tais abordagens objetivaram inferir ao estudante uma rota de aprendizagem otimizada em termos de recurso ou de tempo.

Ainda nos trabalhos mostrados na Tabela 1, em [Torres and Guzmán-Luna 2015], o estilo de aprendizagem é explorado por meio do inventário de Honey-Alonso [Alonso et al. 1999] com o objetivo de subsidiar a identificação da melhor composição de OA para otimizar a rota de aprendizagem do estudante. Em [Marinov and Valova 2016], o perfil do estudante é definido por meio da identificação do conhecimento do estudante acerca do conteúdo abordado, com o intuito de lhe prover problemas conforme com suas habilidades e preferências.

Nos trabalhos supracitados, observa-se uma predominância de modelos do estudante que utilizam estilos de aprendizagem. Neste contexto, sugere-se a investigação de outras características que melhor refinem tais modelos, de maneira a proporcionar impactos positivos na utilização da técnica de PA para recomendação de ações pedagógicas. Isso é interessante pois, o conhecimento acerca do mundo no qual o planejador atuará, é essencial para a otimização de sua atuação [Nau et al. 2004].

No estudo apresentado em [Entwistle and McCune 2004], observa-se a proposição do Approaches and Study Skills Inventory for Students (ASSIST), cuja finalidade é identificar a diretriz representativa da forma como um estudante aborda o estudo. Desse modo, o estudante é classificado sob as dimensões surface, strategic ou deep. Segundo esse estudo, o estudante classificado na categoria surface, apresenta preferência pelo direcionamento do processo de aprendizagem para os requisitos da avaliação. O estudante cuja categoria seja definida como strategic é motivado pela satisfação pessoal, ou seja, prioriza alcançar os melhores resultados por meio de um estudo organizado e otimizando o tempo. Já o aluno identificado na categoria deep direciona seu estudo para atividades de ensino desafiadoras, ou seja, que objetivam a pesquisa do significado das coisas.

De maneira geral, o ASSIST permite a inferência de como o estudante melhor 
VIII Congresso Brasileiro de Informática na Educação (CBIE 2019)

Anais do XXX Simpósio Brasileiro de Informática na Educação (SBIE 2019)

progride durante o processo de aprendizagem, seja em termos da otimização do tempo, da granularidade de atividades recomendadas ou até mesmo do grau de aprofundamento do conteúdo disponível nestas atividades. Diante disso, esta técnica pode auxiliar na identificação da abordagem de aprendizagem mais adequada ao perfil cognitivo do estudante. É possível estabelecer uma correlação entre a Taxonomia de Bloom e o método ASSIST, conforme relatado em [Brown et al. 2015]. Nesse trabalho, é sugerida uma correlação entre estas técnicas, de modo a possibilitar a identificação do estado cognitivo de estudantes num curso introdutório de química, além de vislumbrar possíveis ações pedagógicas adequadas ao estudante.

Analisando o modelo do estudante sob o ponto de vista da Taxonomia Digital de Bloom, observa-se a necessidade de elementos que subsidiem a identificação sobre onde o estudante se localiza nesta em relação à análise bidimensional apresentada na Tabela 2. Para tanto, informações que representem o conteúdo previamente estudado em que o estudante obteve êxito, são de suma importância. Desse modo, neste trabalho proposto, os resultados obtidos em avaliações são considerados para inferir tal informação.

Diante do cenário exposto, a proposição de um modelo capaz de identificar o perfil cognitivo do estudante com o objetivo de sequenciar ações pedagógicas que o auxiliem no processo de aprendizagem, sugere-se viável. Para tanto, faz-se adequada a seguinte composição: i) características relacionadas às estratégias utilizadas pelo estudante no processo de aprendizagem, conforme propostas pelo ASSIST; ii) estruturação referente ao domínio cognitivo, no qual é possível conduzir o estudante para o desenvolvimento de suas habilidades cognitivas, conforme apresentadas pela TDB.

\section{Recomendação de ações pedagógicas usando PA, TDB e ASSIST}

A viabilidade da utilização de PA para personalização de etapas pedagógicas relacionadas ao processo ensino-aprendizagem são demonstradas nos trabalhos citados na Tabela 1. De maneira geral, a maioria desses trabalhos leva em consideração a adoção da modelagem do estudante baseada em estilos de aprendizagem para recomendação de OA, de modo a comporem um caminho de aprendizagem. Com o intuito de oferecer uma solução focada na recomendação de ações pedagógicas genéricas, neste trabalho proposto, o PA é utilizado para recomendação de ações pedagógicas baseadas na TDB, de modo que o foco das recomendações se concentrem no processo cognitivo do estudante.

Dessa maneira, o modelo do estudante proposto é composto por: i) dados obtidos por meio de AVA, à respeito dos resultados dos estudantes referente à atividades realizadas. Esta informação proporciona a identificação do estado cognitivo do estudante em relação à um conteúdo específico; ii) histórico de realização de atividades, obtido por meio de AVA, com o intuito de auxiliar no processo de seleção de atividades com as quais o estudante mais se sente confortável para realizar, baseado no desempenho obtido. iii) estado cognitivo, determinado pela sua localização na TDB. Esta dimensão fornece subsídios para a seleção do plano de ações mais adequado para o estudante; iv) diretriz representativa da forma como o estudante aborda o estudo, fornecida pelo ASSIST, de modo a subsidiar a tomada de decisão quanto à granularidade das atividades recomendadas, de acordo com a classificação do estudante.

Um exemplo de utilização desse modelo pode ser aplicado ao conteúdo Árvore Binária de Busca, numa disciplina Estruturas de Dados num curso de Ciência da 
VIII Congresso Brasileiro de Informática na Educação (CBIE 2019)

Anais do XXX Simpósio Brasileiro de Informática na Educação (SBIE 2019)

Computação, por exemplo. Assim, um Estudante $X$ poderia se encontrar na seguinte situação: Segundo resultados em avaliações diagnósticas para esse conteúdo, sua posição na Tabela 2 seria lembrar/factual, seu objetivo seria criar/metacognitivo e sua classificação segundo o ASSIST seria surface. Nesse cenário, o sistema proposto deve priorizar o desenvolvimento cognitivo gradual do estudante em relação ao conteúdo, por meio do sequenciamento de ações pedagógicas menores e menos complexas. Observa-se também que a otimização da quantidade de ações não deve uma prioridade em detrimento do progresso gradual do estudante. Caso esse mesmo estudante tivesse sua classificação strategic, segundo o ASSIST, o sistema proposto priorizaria a otimização do sequenciamento de ações pedagógicas, reduzindo o tempo para isso. Assim o estudante não necessariamente precisaria progredir sequencialmente em cada uma das etapas das dimensões Processo Cognitivo e Conhecimento da TDB.

Além do sequenciamento das ações pedagógicas, outra etapa importante é a seleção de atividades para o estudante. Conforme apresentado na TDB, diversas atividades podem compor cada uma das categorias das dimensões no domínio cognitivo. Esta seleção pode ser feita, por exemplo, com base no histórico do estudante em relação aos tipos de atividade que esse mais obteve êxito, tornando o processo de aprendizagem mais confortável. Observa-se ainda que a atualização do modelo do estudante é realizada à medida que esse interage com as atividades propostas, proporcionando assim, a adaptação do planejamento às próximas etapas.

Neste trabalho proposto, optou-se pela utilização do Planejamento Baseado em Casos (PBC), que faz uso de soluções anteriormente identificadas e armazenadas, de modo a contribuir com a solução do novo problema. Assim, o PBC é utilizado com base na similaridade entre os modelos dos estudantes. Nesse tipo de abordagem, prioriza-se a busca por um plano que se adéque aos objetivos do estudante, em detrimento da realização do planejamento desde o início, com o intuito de diminuir o custo computacional desta solução. Caso um plano similar não seja encontrado ou não atenda às necessidades do estudante, o planejamento é realizado desde o início [Garrido et al. 2016].

Objetivando a otimização da quantidade de atividades, nota-se que o planejador oferece a solução com menor quantidade de atividades, mesmo havendo outras sequências maiores que levavam ao mesmo objetivo. Na Tabela 3 são apresentados os respectivos estados iniciais e objetivos para dois estudantes, num cenário simulado, bem como suas classificações considerando o ASSIST.

Tabela 3. Definição de objetivos para os estudantes no modelo proposto.

\begin{tabular}{llll}
\hline Estudante & ASSIST & Estado Inicial & Objetivo \\
\hline Estudante_A & surface & Lembrar_1 & Aplicar_4 \\
\hline Estudante_B & strategic & Lembrar_1 & Aplicar_4 \\
\hline
\end{tabular}

A definição dos objetivos e o planejamento das ações pedagógicas para os estudantes listados na Tabela 3 devem considerar o modelo de cada estudante, inicialmente tendo como parâmetro principalmente seus resultados referente à identificação do estado cognitivo segundo a TDB e sua classificação segundo o ASSIST. Por exemplo, considerando o Estudante A classificado como surface, deve-se ter como premissa a definição de ações menores e geralmente de baixa complexidade. 
VIII Congresso Brasileiro de Informática na Educação (CBIE 2019)

Anais do XXX Simpósio Brasileiro de Informática na Educação (SBIE 2019)

oferta automatizada de personalização de etapas do processo pedagógico ao estudante, podendo levar em consideração restrições de recursos ou de tempo, por exemplo.

Outro fator importante é a modelagem das ações pedagógicas sob a perspectiva do processo educacional para além de simplesmente um conteúdo específico. Nesse sentido, a TDB preenche esta lacuna, pois associa ações pedagógicas genéricas à elementos digitais amplamente disseminados. Com isso, esta abordagem apresentada tem como diferencial das demais a proposição de ações pedagógicas genéricas, a partir de metadados de AVA associados ao perfil cognitivo oferecido pelo ASSIST. Assim, compõe-se um modelo para recomendação de ações pedagógicas que subsidiam a tomada de decisão sobre a seleção de atividades que contribuem para a evolução do estado cognitivo do estudante.

Como proposta para evolução deste trabalho, sugere-se a integração do modelo de PA baseado em TDB e guiado pelo ASSIST, a um AVA, com o objetivo de investigar o impacto de metadados como parte dos elementos decisórios para a seleção e recomendação de atividades a fim de otimizar o processo de aprendizagem. Diante das técnicas de PA relatadas anteriormente, sugere-se também uma investigação sobre a eficiência destas para estudantes classificados em cada uma das dimensões apresentadas pelo ASSIST.

\section{Agradecimentos}

Os autores agradecem ao Instituto Federal de Educação, Ciência e Tecnologia Goiano (IF Goiano) e à Universidade Federal de Uberlândia (UFU), pelo apoio à esta pesquisa.

\section{Referências}

Alonso, C., Gallego, D., and Honey, P. (1999). Los estilos de aprendizaje. procedimientos de diagnóstico y mejora (4ta edición). bilbao: Ediciones mensajero.

Brown, S., White, S., Wakeling, L., and Naiker, M. (2015). Approaches and study skills inventory for students (assist) in an introductory course in chemistry. Journal of University Teaching \& Learning Practice.

Caputi, V. and Garrido, A. (2015). Student-oriented planning of e-learning contents for moodle. Journal of Network and Computer Applications, 53:115 - 127.

Churches, A. (2010). Bloom's digital taxonomy.

Costa, N., Pereira Junior, C., Araújo, R., and Fernandes, M. (2019). Application of ai planning in the context of e-learning. In International Conference on Advanced Learning Technologies (ICALT), page 57.

Dantas, A. C., de Melo, S., Fernandes, M., Lima, L., and do Nascimento, M. Z. (2018). Recomendação de estratégias pedagógicas através de emoções, perfis de personalidade e inteligências múltiplas utilizando raciocínio baseado em casos. In Brazilian Symposium on Computers in Education (Simpósio Brasileiro de Informática na EducaçãoSBIE), volume 29, page 1213.

de Paiva, R. A. and Padilha, M. A. S. (2012). A webquest e a taxonomia digital de bloom como uma nova coreografia didática para a educação online. Revista Brasileira de Ensino de Ciência e Tecnologia, 5(1).

Entwistle, N. and McCune, V. (2004). The conceptual bases of study strategy inventories. Educational Psychology Review, 16(4):325-345. 
VIII Congresso Brasileiro de Informática na Educação (CBIE 2019)

Anais do XXX Simpósio Brasileiro de Informática na Educação (SBIE 2019)

Felder, R. M., Silverman, L. K., et al. (1988). Learning and teaching styles in engineering education. Engineering education, 78(7):674-681.

Garrido, A. and Morales, L. (2014). E-learning and intelligent planning: Improving content personalization. IEEE Revista Iberoamericana de Tecnologias del Aprendizaje, 9(1):1-7.

Garrido, A., Morales, L., and Serina, I. (2016). On the use of case-based planning for e-learning personalization. Expert Systems with Applications, 60:1-15.

Garrido, A. and Onaindia, E. (2013). Assembling learning objects for personalized learning: An ai planning perspective. IEEE Intelligent Systems, 28(2):64-73.

Haendchen Filho, A., Tomazoni, E. K., Paza, R., Perego, R., and Raabe, A. (2018). Bloom's taxonomy-based approach for assisting formulation and automatic short answer grading. In Brazilian Symposium on Computers in Education (Simpósio Brasileiro de Informática na Educação-SBIE), volume 29, page 238.

Krathwohl, D. R. (2002). A revision of bloom's taxonomy: An overview. Theory Into Practice, 41(4):212-218.

Leu, D. J., Kinzer, C. K., Coiro, J., Castek, J., and Henry, L. A. (2017). New literacies: A dual-level theory of the changing nature of literacy, instruction, and assessment. Journal of Education, 197(2):1-18.

Limongelli, C. and Sciarrone, F. (2014). Fuzzy student modeling for personalization of elearning courses. In Zaphiris, P. and Ioannou, A., editors, Learning and Collaboration Technologies. Designing and Developing Novel Learning Experiences, pages 292-301, Cham. Springer International Publishing.

Marinov, M. and Valova, I. (2016). Application of planning techniques in knowledgemanaged tutoring systems. In 2016 15th International Conference on Information Technology Based Higher Education and Training (ITHET), pages 1-5.

Nau, D., Ghallab, M., and Traverso, P. (2004). Automated Planning: Theory \& Practice. Morgan Kaufmann Publishers Inc., San Francisco, CA, USA.

Ohler, J. B. (2013). Digital storytelling in the classroom: New media pathways to literacy, learning, and creativity. Corwin Press.

Pireva, K. and Kefalas, P. (2018). A recommender system based on hierarchical clustering for cloud e-learning. Intelligent Distributed Computing XI, 53:235 - 245.

Sanchez Nigenda, R., Maya Padrón, C., Martínez-Salazar, I., and Torres-Guerrero, F. (2017). Design and evaluation of planning and mathematical models for generating learning paths. Computational Intelligence, 34(3):821-838.

Torres, I.-D. and Guzmán-Luna, J. (2015). Reactive planning to compose learning routes in uncertain environments. In New Trends in Networking, Computing, E-learning, Systems Sciences, and Engineering, pages 101-108. Springer.

Xu, D., Huang, W. W., Wang, H., and Heales, J. (2014). Enhancing e-learning effectiveness using an intelligent agent-supported personalized virtual learning environment: An empirical investigation. Information \& Management, 51(4):430 - 440. 